\title{
A Validation Study of the Brief Alcohol Use Disorder Identification Test (AUDIT): A Brief Screening Tool Derived from the AUDIT

\author{
Kyungseop So, Eunju Sung ${ }^{1, *}$
}

Total Healthcare Center, ${ }^{1}$ Department of Family Medicine, Kangbuk Samsung Hospital, Sungkyunkwan University School of Medicine, Seoul, Korea

Background: The prevalence of alcohol use disorder (AUD) is very high in Korea. To identify AUD in the busy practice setting, brevity of screening tools is very important. We derived the brief Alcohol Use Disorders Identification Test (AUDIT) and evaluated its performance as a brief screening test.

Methods: One hundred male drinkers from Kangbuk Samsung Hospital primary care outpatient clinic and psychiatric ward for alcoholism treatment completed questionnaires including the AUDIT, cut down, annoyed, guilty, eye-opener (CAGE), and National Alcoholism Screening Test (NAST) from April to July, 2007. AUD (alcohol abuse and dependence), defined by a physician in accordance with Diagnostic and Statistical Manual of Mental Disorders-IV, was used as a diagnostic criteria. To derive the brief AUDIT, factor analysis was performed using the principal component extraction method with a varimax rotated solution. Receiver operating characteristic (ROC) curve analysis was performed to investigate the discrimination ability of the brief AUDIT. Areas under the ROC curve were compared performance of screening questionnaires with $95 \%$ confidence intervals.

Results: The derived brief AUDIT consists of 4 items: frequency of heavy drinking (item 3), impaired control over drinking (item 4), increased salience of drinking (item 5), and alcohol-related injury (item 9). Brief AUDIT exhibited an AUD screening accuracy better than CAGE, and equally to that of NAST. Areas under the ROC curves were $0.87(0.80-0.94), 0.76$ (0.66-0.85), and $0.81(0.73-0.90)$ for the brief AUDIT, CAGE, and NAST for AUD, and 0.97 (0.95-0.99), 0.93 (0.88-0.98) and 0.93 (0.88-0.98) for alcohol dependence.

Conclusion: The new brief AUDIT seems to be effective in detecting male AUD in the primary care setting in Korea. Further evaluation for women and different age groups is needed.

Keywords: Alcohol; Mass Screening; Primary Health Care

\section{INTRODUCTION}

Received: October 30, 2012, Accepted: December 28, 2012

${ }^{*}$ Corresponding Author: Eunju Sung

Tel: +82-2-2001-2277, Fax: +82-2-2001-1280

E-mail: eunjusung68@gmail.com

Korean Journal of Family Medicine

Copyright $(c) 2013$ The Korean Academy of Family Medicine

(a) This is an open-access article distributed under the terms of the Creative Commons Attribution Non-Commercial License (http://creativecommons.org/licenses/by-nc/3.0) which permits unrestricted noncommercial use, distribution, and reproduction in any medium, provided the original work is properly cited.
The proportion of Korean adults who drink alcohol is among the world's highest and is increasing-from $45.8 \%$ in 1989 to $59.2 \%$ in $2005 .{ }^{1,2)}$ According to the Korean national health and nutrition examination survey, the overall age-adjusted prevalence of alcohol use disorder (AUD: alcohol abuse and alcohol dependence) in 2009 was $38.8 \%$. Men were about 7 times as likely as women to meet the criteria for AUD. ${ }^{3)} \mathrm{A}$ large portion of Korean men drink alcohol excessively. In addition, the prevalence 
of AUD is much higher in Korea compared to other countries. ${ }^{46)}$

Alcohol is implicated in a wide variety of diseases, disorders, and injuries, as well as many social and legal problems. The majority of excessive drinkers are undiagnosed. Often they present with symptoms or problems that would not normally be linked to their drinking. Brief intervention is a low-cost, effective preventive measure for heavy drinkers in outpatient settings. Therefore, screening heavy drinkers who need brief intervention is very important, especially in primary care. ${ }^{7)}$

Cut down, annoyed, guilty, eye-opener (CAGE), the Michigan Alcoholism Screening Test (MAST), National Alcoholism Screening Test (NAST), and Alcohol Use Disorders Identification Test (AUDIT) are well known tools to screen for AUD. CAGE is the briefest effective screening test for lifetime alcohol abuse and/or dependence but it is insensitive for detecting heavy drinking and does not distinguish between active and past AUD. ${ }^{8)}$ MAST was devised as a structured interview instrument, and consists of 25 questions. ${ }^{9)}$ NAST (Alcoholism Screening Test of Seoul National Mental Hospital) was devised to screen Korean alcoholism. It was originally designed with 12 items to screen AUD in those who need psychiatric intervention in psychiatric inpatients. ${ }^{10)}$

The AUDIT is the first screening test designed specifically for use in primary care settings, and validated in Korea. ${ }^{11)}$ The AUDIT was developed to screen for excessive drinking and in particular to help practitioners identify people who would benefit from reducing or ceasing drinking. ${ }^{12,13)}$ Although its sensitivity, specificity, and other psychometric properties compare at least equally or exceed those of other screening instruments, the length of the AUDIT may preclude its use in settings where brevity is critical, such as in a primary care setting or emergency room. For such purposes, abbreviated versions of the AUDIT exist such as AUDIT-consumption (C), AUDIT-Primary Care, AUDIT-3, Rapid Alcohol Problems Screen (RAPS), RAPS4-quantityfrequency, and Alcohol Screening Test (FAST). ${ }^{14-18)}$ Among them, AUDIT-C, which consists of the first 3 items of the AUDIT (the consumption factor items), has generated wide research interest. ${ }^{19)}$ Recently, AUDIT-C appeared to perform better than AUDIT-3 in the Korean primary care setting, but validated only against the full AUDIT rather than against an independent, formal alcohol diagnosis or hazardous drinking criterion. ${ }^{20)}$

As alcohol drinking behavior depends heavily on culture and ethnicity, we tried to derive a Korean short version of the
AUDIT (brief AUDIT). We evaluated the brief AUDIT as a quick screening test for AUD, and compared the performance of the brief AUDIT with that of other validated alcohol screening questionnaires.

\section{METHODS}

\section{Study Population}

This study was conducted at Kangbuk Samsung Hospital primary care outpatient clinic and psychiatric ward for alcoholism treatment. The study population was 85 male drinkers from the primary care setting, and 15 male alcohol dependency patients from a psychiatric hospital from April to July, 2007, who consented with the study. The questionnaire was filled out by patients, and then their physician ( 1 psychiatrist and 4 family physicians) defined AUD in accordance with the Diagnostic and Statistical Manual of Mental Disorders-IV without knowing the results of the questionnaire.

\section{Measures}

The questionnaire consisted of demographic factors, AUDIT, CAGE, and NAST. The AUDIT core questionnaire consists of 10 items: three questions on quantity and frequency of drinking, three items on alcohol dependence and four questions on problems caused by drinking. Weighted scoring with respect to the frequency or the time of occurrence results in total scores ranging from 0 to $40 .^{12)}$

The CAGE is an acronym based on its four items: cut down on drinking, annoyed by criticism, guilty feelings, and eye opener. NAST consists of 12 items: easily feels self-pity, prefers drinking alone, drinks in the morning, feels a compelling desire to drink continuously, has difficulty resisting the impulse to drink, has recent amnesia about events during drunkenness, feels drinking is harmful to social life, has had damage to occupation, has a spouse about to leave, suffers from alcohol withdrawal symptom, suffers from serious alcohol withdrawal symptom, has had treatment for alcohol related problems. ${ }^{10)}$

\section{Statistical Analysis}

To derive brief AUDIT, factor analysis was performed using the principal component extraction method with a varimax 
rotated solution. One-way analysis of variance was used to compare brief AUDIT between groups (normal, alcohol abuse, and alcohol dependence). Pearson correlation analysis was used to assess the relationship between tests.

Screening performance was evaluated by means of sensitivity, specificity, positive likelihood ratio, negative likelihood ratio and areas under receiver operating characteristics (ROCs). ROC curves plotted sensitivity versus 1 -specificity, and areas under the curves and their $95 \%$ confidence intervals were determined. The area under an ROC curve (AUROC) is a measure of a screening test's overall screening performance taking into account all possible screening thresholds. Areas under ROC curves closer to 1.0 indicate a better performance and an area under a curve of 0.5 yields no useful information. Cronbach's alpha was calculated to assess internal consistency of brief AUDIT. All statistics were performed using the SPSS ver. 15.0 (SPSS Inc., Chicago, IL, USA).

Table 1. Demographic characteristics of study subjects

\begin{tabular}{|c|c|c|}
\hline \multicolumn{2}{|c|}{ Characteristics } & \multirow{2}{*}{$\begin{array}{c}\text { Number } \\
10\end{array}$} \\
\hline Age (y) & $<29$ & \\
\hline & $30-39$ & 19 \\
\hline & $40-49$ & 39 \\
\hline & $50-59$ & 18 \\
\hline & $\geq 60$ & 14 \\
\hline \multirow[t]{4}{*}{ Education } & Elementary school & 6 \\
\hline & Middle school & 11 \\
\hline & High school & 25 \\
\hline & University & 58 \\
\hline \multirow[t]{3}{*}{ Occupation } & White collar & 44 \\
\hline & Blue collar & 22 \\
\hline & Others & 34 \\
\hline \multirow[t]{4}{*}{ Marital status } & Married & 69 \\
\hline & Unmarried & 19 \\
\hline & Divorced & 11 \\
\hline & Separation by death & 1 \\
\hline Total & & 100 \\
\hline
\end{tabular}

\section{RESULTS}

\section{Characteristics of Study Population}

Among subjects in the study population, 49 (49\%) were defined as the normal group, 27 (27\%) as abusive of alcohol, and $24(24 \%)$ as alcohol-dependent. Mean age was $45.4 \pm 11.0$ (standard deviation) years. Table 1 shows the demographic characteristics of the study population.

\section{Development of Brief Alcohol Use Disorders Identification Test}

Factor analysis was conducted to select questions from 10 items. Principal component extraction method identified 2 factors, and they accounted for $65 \%$ of total variance. The two factors and their components are presented in Table 2. One was alcohol-dependency and related problems (impaired control over drinking, increased salience of drinking, morning drinking, guilt after drinking, blackouts, alcohol-related injuries, and others concerned about drinking), and the other was consumption (frequency, typical quantity, and frequency of heavy drinking). To derive items for brief AUDIT, two criteria were adjusted. First, items with a factor loading value over 0.8 were selected. Items number 2, 3, 4, 5, and 9 were selected. Items 4, 5, and 9 belonged to factor one, and 2 and 3 to factor two (Table 2). Second, items which do not attribute Cronbach's alpha value were excluded. The AUDIT showed Cronbach's alpha of 0.872. After exclusion

Table 2. Result of factor analysis for Alcohol Use Disorders Identification Test*

\begin{tabular}{cccl}
\hline Item & Factor 1 & Factor 2 & \multicolumn{1}{c}{ Content } \\
\hline 5 & 0.870 & 0.158 & Increased salience of drinking \\
9 & 0.818 & 0.000 & Alcohol-related injuries \\
4 & 0.802 & 0.312 & Impaired control over drinking \\
7 & 0.768 & 0.177 & Guilt after drinking \\
6 & 0.676 & 0.122 & Morning drinking \\
8 & 0.629 & 0.444 & Blackouts \\
10 & 0.494 & 0.452 & Others concerned about drinking \\
2 & -0.019 & 0.843 & Typical quantity \\
3 & 0.307 & 0.806 & Frequency of heavy drinking \\
1 & 0.186 & 0.797 & Frequency of drinking \\
\hline
\end{tabular}

*Values are factor loading. 
of item 2, alpha was 0.875 (Table 3). Item 2 was near the typical quantity. Among consumption items, it seemed frequency of heavy drinking (item 3 ) perform better than the typical quantity (item 2). Item 3 is the one of AUDIT-3. So, item 3 was left for the brief AUDIT. The final items for the brief AUDIT were frequency of heavy drinking (item 3), impaired control over drinking (item 4), increased salience of drinking (item 5), and alcohol-related injury (item 9). The score system was applied the same as in AUDIT, total scores ranging from 0 to 16 .

Cronbach's alpha was calculated to assess internal consistency of the brief AUDIT. The brief AUDIT showed an alpha value of 0.82. The Cronbach's alpha of NAST, CAGE, and original AUDIT were $0.86,0.69$, and 0.87 each. The internal consistency analysis yielded high Cronbach's alpha values for the full and brief AUDIT and NAST. CAGE was not sufficiently consistent compared to that of other questionnaires.

\section{Performance of Brief Alcohol Use Disorders Identification Test \\ The brief AUDIT showed a high correlation with the 10-item}

original AUDIT, with a coefficient of 0.93 , good correlation with NAST of 0.80 , and CAGE of $0.76(\mathrm{P}<0.05)$. The brief AUDIT discriminated AUD diagnosis as presented in Table 4. The mean of each group was 3.8, that of alcohol abuse 6.0, and alcohol dependence 11.9. The difference was statistically significant $(\mathrm{F}=$ 93.473, $\mathrm{df}=2, \mathrm{P}<0.001$ ) (Table 4). ROC curves are depicted in the Figure 1, and the AUC for each ROC curve was calculated. Sensitivity, specificity, positive likelihood ratio, and negative likelihood ratio by cut-off points are presented in Table 5 .

Optimum cut-off value was identified with the ROC curve. The brief AUDIT threshold that simultaneously maximized sensitivity and specificity for detecting AUD was $\geq 6$ (sensitivity $83.3 \%$, specificity $96.1 \%$ ), and for alcohol dependence was $\geq 10$ (sensitivity $83.3 \%$, specificity $96.1 \%$ ) (Table 5). As we included hospitalized AUD patients in the study, it is possible that our values are higher than in the primary care setting. The purpose of the brief AUDIT is to detect AUD in a short time. As maximizing sensitivity is the best policy for screening disease like AUDs, we chose a cutoff point of $\geq 5$ (sensitivity $80.4 \%$, positive likelihood ratio [+LR] 2.62) for AUDs, and $\geq 9$ (sensitivity $87.5 \%,+\mathrm{LR}$

Table 3. Item analysis of Alcohol Use Disorders Identification Test

\begin{tabular}{rcccc}
\hline Item & $\begin{array}{c}\text { Scale variance if item } \\
\text { deleted }\end{array}$ & $\begin{array}{c}\text { Corrected item-total } \\
\text { correlation }\end{array}$ & $\begin{array}{c}\text { Squared multiple } \\
\text { correlation }\end{array}$ & \multicolumn{2}{c}{ Cronbach's alpha if item } \\
deleted
\end{tabular}

Table 4. Comparison of brief AUDIT scores between normal, alcohol abuse, and $\mathrm{AD}$

\begin{tabular}{ccccccc}
\hline & Normal $(\mathrm{n}=49)$ & Alcohol abuse $(\mathrm{n}=27)$ & $\mathrm{AD}(\mathrm{n}=24)$ & $\mathrm{F}$ & df & P-value* \\
\hline Mean $\pm \mathrm{SD}$ & $3.8 \pm 2.1$ & $6.0 \pm 2.8$ & $11.9 \pm 2.3$ & 93.47 & 2 & 0.000 \\
\hline
\end{tabular}

AUDIT: Alcohol Use Disorders Identification Test, $\mathrm{AD}$ : alcohol dependence.

*Analysis of variance and post-hoc. 
9.51) for alcohol dependence (Table 5).

The brief AUDIT's AUROC curve for AUD was $0.87 \pm 0.04$ (standard error $[\mathrm{SE}]$ ). The brief AUDIT had a greater AUROC curve than either CAGE $0.76 \pm 0.05$ (SE), or NAST $0.82 \pm 0.04$ (SE) for alcohol abuse.

The brief AUDIT's AUROC curve for alcohol dependence was $0.97 \pm 0.01(\mathrm{SE})$. It was greater than that of NAST $0.93 \pm 0.02$

A

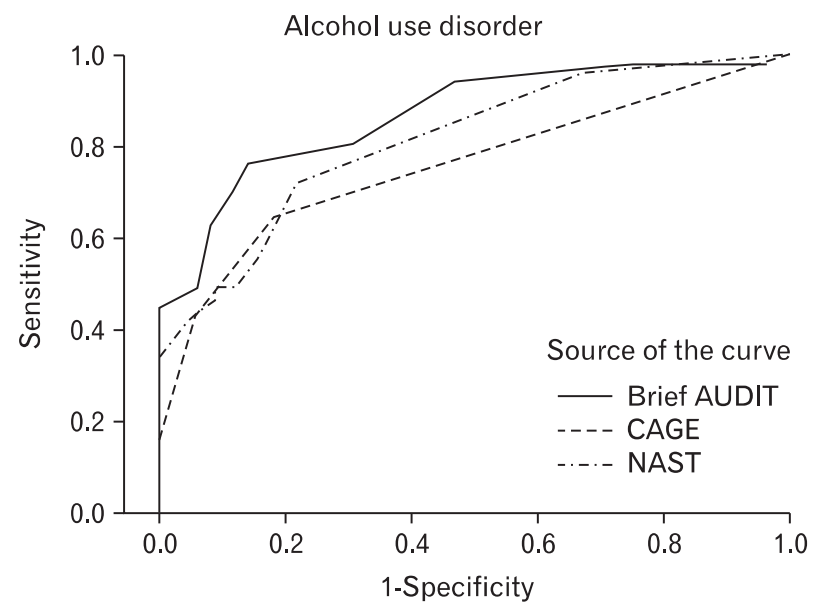

(SE) or CAGE $0.93 \pm 0.03$ (SE) (Table 6).

\section{DISCUSSION}

To our knowledge, this is the first study to derive a short form of the AUDIT (brief AUDIT) and compare the performance of

B

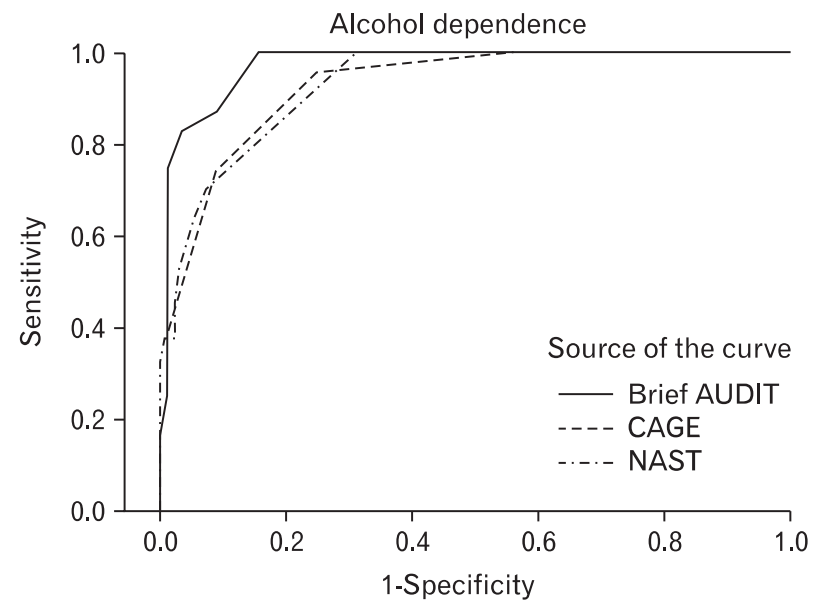

Figure 1. Receiver operating characteristic curves of the brief Alcohol Use Disorders Identification Test (AUDIT), National Alcoholism Screening Test (NAST), and cut down, annoyed, guilty, eye-opener (CAGE) for alcohol use disorder (A) and alcohol dependence (B).

Table 5. Performance of brief AUDIT questionnaire for detecting any AUDs or AD

\begin{tabular}{cccccc}
\hline & Cut-off score (AUDs) & Sensitivity (\%) & Specificity (\%) & +LR & -LR \\
\hline \multirow{2}{*}{ AUDs } & $\geq 5$ & 80.4 & 69.4 & 2.62 & 0.28 \\
& $\geq 6$ & 76.5 & 85.7 & 5.34 & 0.27 \\
& $\geq 7$ & 66.7 & 89.8 & 6.54 & 0.37 \\
$\mathrm{AD}$ & $\geq 9$ & 87.5 & 90.8 & 9.51 & 0.13 \\
& $\geq 10$ & 83.3 & 96.1 & 21.1 & 0.17 \\
& $\geq 11$ & 75.0 & 98.7 & 56.8 & 0.25 \\
\hline
\end{tabular}

AUDIT: Alcohol Use Disorders Identification Test, AUD: alcohol use disorders, AD: alcohol dependence, +LR: positive likelihood ratio, -LR: negative likelihood ratio.

Table 6. AUCs comparison of brief AUDIT with CAGE and NAST

\begin{tabular}{|c|c|c|c|c|c|c|}
\hline \multirow{2}{*}{ Group } & \multicolumn{2}{|c|}{ Brief AUDIT } & \multicolumn{2}{|c|}{ CAGE } & \multicolumn{2}{|c|}{ NAST } \\
\hline & AUC & $95 \% \mathrm{CI}$ & AUC & $95 \% \mathrm{CI}$ & AUC & $95 \%$ CI \\
\hline Any AUDs & 0.868 & $0.798-0.939$ & 0.756 & $0.66-0.852$ & 0.814 & $0.732-0.896$ \\
\hline $\mathrm{AD}$ & 0.972 & $0.945-0.999$ & 0.928 & $0.878-0.979$ & 0.928 & $0.879-0.977$ \\
\hline
\end{tabular}

AUC: area under the curve, AUDIT: Alcohol Use Disorders Identification Test, CAGE: cut down, annoyed, guilty, eye-opener, NAST: National Alcoholism Screening Test, CI: confidence interval, AUD: alcohol use disorders, AD: alcohol dependence. 
it with that of well known AUD screening questionnaires, CAGE, and NAST, in the primary care setting in Korea.

The new brief AUDIT consists of 4 items: frequency of heavy drinking (item 3), impaired control over drinking (item 4), increased salience of drinking (item 5), and alcohol-related injury (item 9), with a scoring range from 0 to 16. The brief AUDIT discriminated AUD very well. The cut-off point was 5 to detect any AUD (sensitivity $80.4 \%$, specificity $69.4 \%$ ), and 9 for alcohol dependence (sensitivity $87.5 \%$, specificity $90.8 \%$ ). The brief AUDIT's AUROC curve for alcohol-use disorder was $0.87 \pm 0.04$ (SE), and for alcohol dependence was $0.97 \pm 0.01$ (SE). The brief AUDIT had a greater AUROC curve than either CAGE $0.76 \pm$ 0.05 (SE), or NAST $0.82 \pm 0.04$ (SE) for Any AUD, and greater than either NAST $0.93 \pm 0.02$ (SE) or CAGE $0.93 \pm 0.03$ (SE) for alcohol dependence. As we targeted primary outpatient setting, we put weight for higher sensitivity to detect any AUD and higher specificity for alcohol dependence.

AUDIT was originally designed for practitioners to screen problem drinkers, i.e., drinking problems less severe than diagnosable disorders of AUD as well as AUD. The full 10item AUDIT with its multidimensional scoring for each item has been called "cumbersome" for use in some settings where rapidity of scoring, as well as accuracy, is important. ${ }^{21)}$ Many abbreviated versions of AUDIT were generated and tested to meet this practical need. AUDIT-C, the most commonly tested derived AUDIT, consists of the first 3 items of the AUDIT (the consumption factor items). AUDIT-C was reported useful to screen problem drinkers, and AUD in western countries. FAST is a 4-item scale consisting of item 3 (modified for men by increasing the number of drinks from 1 occasion to 8 ) as well as items 5, 8, and 10 from the original AUDIT. Unfortunately, these researchers validated the abbreviated scale only against the full AUDIT rather than also against an independent, formal alcohol diagnosis or hazardous drinking criterion. ${ }^{22)}$

Drinking behavior of the western population may be quite different from that of Korea. Heavy drinking is very popular because alcohol is accepted generously and quite often, and is socially forced in work-related gatherings, especially for men. This consumption factor might not be enough to discriminate AUD, however it is the main reason we decided to derive a short version for Koreans rather than using the existing one. We compared the performance of the brief AUDIT with the AUDIT-C and FAST.
The AUROC against AUD was 0.868 for the brief AUDIT, 0.739 for the AUDIT-C, and 0.898 for FAST. The AUROC for alcohol dependence was 0.972 for the brief AUDIT, 0.664 for the AUDIT-C, and 0.942 for FAST (data not presented). The performance of the brief AUDIT was about equal to FAST, and better than the AUDIT-C. The performance of the AUDIT-C to detect AUD against formal alcohol diagnosis need to be further evaluated for Koreans.

The AUDIT questionnaire is measured consistently over time and is particularly useful in detecting recent problem drinking. Investigators in a six-country World Health Organization project developed the AUDIT to screen patients in primary health care settings for hazardous or harmful drinking and serve as a basis for discussion with patents in brief therapy. ${ }^{12)}$ Hazardous drinkers are individuals at high risk for alcohol-related damage to physical or psychological health; harmful drinkers already experience such problems. ${ }^{23)}$ The original cut-off point to detect hazardous drinker is 8 , yielding a sensitivity of $0.90 \mathrm{~s}$, and specificity of $0.80 \mathrm{~s}$. To find the hazardous drinking cut-off point, the gold standard criteria need to be multidimensional: heavy consumption (i.e., 14 or more drinks per week), laboratory results or clinical diagnosis like alcoholic hepatitis, as well as diagnosis of AUD. The performance of AUDIT against those criteria needs to be evaluated further to make the AUDIT more practical, and at the same time, accurate for Koreans. Also, further study to evaluate the performance of the abbreviated version of the AUDIT against other criteria than AUD might be useful to detect problem drinkers.

Limitations of this study were as follows. Firstly, the study subjects were not representative of the entire population. Women were excluded, and the age range of study subjects was 32 to 63 . Cut-off points could be different for different populations, as alcohol affects women differently. Women were excluded because alcohol screening questionnaires function differently in men and women ${ }^{19)}$ and we could not recruit an adequate number of women through which to base any conclusions regarding questionnaire performance. Also we collected $15 \%$ of study subjects from the psychiatric ward and not only from primary outpatient clinics for the same reason. Secondly, performance of the brief AUDIT on criteria of problem drinking was not measured as mentioned above. Thirdly, test-retest was not evaluated. Lastly, we used the same population to derive and validate the brief AUDIT. 
The brief AUDIT was short and performed well to screen alcohol-use disorders in middle-aged men. It will facilitate screening for alcohol problems where significant constraints on time exist. Further research to evaluate performance of the brief AUDIT against criteria of problem drinking in large populations including other age groups and women are needed to verify its effectiveness.

\section{CONFLICT OF INTEREST}

No potential conflict of interest relevant to this article was reported.

\section{REFERENCES}

1. Koh KH. Estimates of alcohol consumption based on pure alcohol content and their comparison with data of other countries. Health Welf Policy Forum 2000;6:77-86.

2. Ministry of Health and Welfare. The Third Korea National Health and Nutrition Examination Survey (KNHANES III). Seoul: Ministry of Health and Welfare; 2005.

3. Park SH, Kim CH, Kim DJ, Suk KT, Park HY, Lee JG, et al. Secular trends in prevalence of alcohol use disorder and its correlates in Korean adults: results from Korea National Health and Nutrition Examination Survey 2005 and 2009. Subst Abus 2012;33:327-35.

4. Lee CK, Kwak YS, Yamamoto J, Rhee H, Kim YS, Han JH, et al. Psychiatric epidemiology in Korea. Part I: Gender and age differences in Seoul. J Nerv Ment Dis 1990;178:242-6.

5. Lee CK, Kwak YS, Yamamoto J, Rhee H, Kim YS, Han JH, et al. Psychiatric epidemiology in Korea. Part II: Urban and rural differences. J Nerv Ment Dis 1990;178:247-52.

6. Min S, Noh S, Shin J, Ahn JS, Kim TH. Alcohol dependence, mortality, and chronic health conditions in a rural population in Korea. J Korean Med Sci 2008;23:1-9.

7. Babor TF, Higgins-Biddle JC, Saunders JB, Monteiro MG; World Health Organization. AUDIT: the alcohol use disorders identification test: guidelines for use in primary health care. 2nd ed. Geneva: World Health Organization;
2001.

8. Mayfield D, McLeod G, Hall P. The CAGE questionnaire: validation of a new alcoholism screening instrument. Am J Psychiatry 1974;131:1121-3.

9. Selzer ML. The Michigan alcoholism screening test: the quest for a new diagnostic instrument. Am J Psychiatry 1971;127: 1653-8.

10. Kim KB, Hahn KS, Lee JK, Rhee MK, Kim YK, Kim CK. The preliminary study of the Korean alcoholism screening test (III). J Korean Neuropsychiatr Assoc 1991;30:569-81.

11. Kim JS, Oh MK, Park BK, Lee MK, Kim GJ. Screening criteria of alcoholism by alcohol use disorders identification test (AUDIT) in Korea. J Korean Acad Fam Med 1999;20: 1152-9.

12. Allen JP, Litten RZ, Fertig JB, Babor T. A review of research on the Alcohol Use Disorders Identification Test (AUDIT). Alcohol Clin Exp Res 1997;21:613-9.

13. Saunders JB, Aasland OG, Babor TF, de la Fuente JR, Grant M. Development of the Alcohol Use Disorders Identification Test (AUDIT): WHO Collaborative Project on Early Detection of Persons with Harmful Alcohol Consumption-II. Addiction 1993;88:791-804.

14. Cherpitel CJ, Bazargan S. Screening for alcohol problems: comparison of the audit, RAPS4 and RAPS4-QF among African American and Hispanic patients in an inner city emergency department. Drug Alcohol Depend 2003;71:27580.

15. Cherpitel CJ, Ye Y, Moskalewicz J, Swiatkiewicz G. Screening for alcohol problems in two emergency service samples in Poland: comparison of the RAPS4, CAGE and AUDIT. Drug Alcohol Depend 2005;80:201-7.

16. Gomez A, Conde A, Santana JM, Jorrin A. Diagnostic usefulness of brief versions of Alcohol Use Disorders Identification Test (AUDIT) for detecting hazardous drinkers in primary care settings. J Stud Alcohol 2005;66:305-8.

17. Gordon AJ, Maisto SA, McNeil M, Kraemer KL, Conigliaro $\mathrm{RL}$, Kelley ME, et al. Three questions can detect hazardous drinkers. J Fam Pract 2001;50:313-20.

18. Piccinelli M, Tessari E, Bortolomasi M, Piasere O, Semenzin M, Garzotto N, et al. Efficacy of the alcohol use disorders identification test as a screening tool for hazardous alcohol 
intake and related disorders in primary care: a validity study. BMJ 1997;314:420-4.

19. Reinert DF, Allen JP. The alcohol use disorders identification test: an update of research findings. Alcohol Clin Exp Res 2007;31:185-99.

20. Seong JH, Lee CH, Do HJ, Oh SW, Lym YL, Choi JK, et al. Performance of the AUDIT Alcohol Consumption Questions (AUDIT-C) and AUDIT-K question 3 alone in screening for problem drinking. Korean J Fam Med 2009;30:695-702.
21. Hearne R, Connolly A, Sheehan J. Alcohol abuse: prevalence and detection in a general hospital. J R Soc Med 2002;95:847.

22. Hodgson R, Alwyn T, John B, Thom B, Smith A. The fast alcohol screening test. Alcohol Alcohol 2002;37:61-6.

23. Allen JP, Reinert DF, Volk RJ. The alcohol use disorders identification test: an aid to recognition of alcohol problems in primary care patients. Prev Med 2001;33:428-33. 